\title{
ESSENTIAL COMMUTATIVITY OF SOME INTEGRAL AND COMPOSITION OPERATORS
}

\author{
ZE-HUA ZHOU ${ }^{凶}$, LIANG ZHANG and HONG-GANG ZENG
}

(Received 2 June 2011)

\begin{abstract}
In general, multiplication of operators is not essentially commutative in an algebra generated by integraltype operators and composition operators. In this paper, we characterize the essential commutativity of the integral operators and composition operators from a mixed-norm space to a Bloch-type space, and give a complete description of the universal set of integral operators. Corresponding results for boundedness and compactness are also obtained.
\end{abstract}

2010 Mathematics subject classification: primary 47B38; secondary 47B33, 45P05, 46E15, 30H30.

Keywords and phrases: essential commutativity, integral-type operators, composition operators.

\section{Introduction}

Let $d A$ denote Lebesgue measure on $\mathbb{D}$, normalized so that $A(\mathbb{D})=1$, where $\mathbb{D}$ is the open unit disk in the complex space $\mathbb{C}$. Let $H(\mathbb{D})$ be the space of all analytic functions on $\mathbb{D}$, and $S(\mathbb{D})$ the collection of all analytic self mappings of $\mathbb{D}$. For $0<p, q<\infty$ and $-1<\gamma<\infty$, the mixed-norm space $H_{p, q, \gamma}=H_{p, q, \gamma}(\mathbb{D})$ consists of all $f \in H(\mathbb{D})$ such that

$$
\|f\|_{H_{p, q, \gamma}}=\left(\int_{0}^{1} M_{p}^{q}(f, r)(1-r)^{\gamma} d r\right)^{1 / q}<\infty
$$

where

$$
M_{p}(f, r)=\left(\frac{1}{2 \pi} \int_{0}^{2 \pi}\left|f\left(r e^{i \theta}\right)\right|^{p} d \theta\right)^{1 / p} .
$$

For $\alpha>0$, recall that the $\alpha$-Bloch space $\mathcal{B}^{\alpha}=\mathcal{B}^{\alpha}(\mathbb{D})$ is the space of all $f \in H(\mathbb{D})$ such that

$$
b_{\alpha}(f)=\sup _{z \in \mathbb{D}}\left(1-|z|^{2}\right)^{\alpha}\left|f^{\prime}(z)\right|<\infty .
$$

The expression $b_{\alpha}(f)$ defines a semi-norm, while the natural norm is given by $\|f\|_{\mathcal{B}^{\alpha}}=$ $|f(0)|+b_{\alpha}(f)$. This norm makes $\mathcal{B}^{\alpha}$ into a Banach space (see, for example [14]). For

The first author was supported in part by the National Natural Science Foundation of China (Grant Nos. 10971153, 10671141).

(c) 2011 Australian Mathematical Publishing Association Inc. 0004-9727/2011 \$16.00 
$\alpha \leq 0$, we still use $\mathcal{B}^{\alpha}$ to stand for the space of all $f \in H(\mathbb{D})$ such that $b_{\alpha}(f)<+\infty$. By the maximum modulus theorem, it is easy to show that $\mathcal{B}^{\alpha}=\mathbb{C}$ for $\alpha<0$.

Every $\varphi \in S(\mathbb{D})$ induces a composition operator $C_{\varphi}$ defined by $C_{\varphi} f=f \circ \varphi$ for $f \in H(\mathbb{D})$. Readers interested in this operator can refer to the papers $[7-9,12,13]$ and the references therein.

If $g \in H(\mathbb{D})$, the integral operators $J_{g}$ and $I_{g}$ are defined by

$$
J_{g} f(z)=\int_{0}^{z} f(\zeta) g^{\prime}(\zeta) d \zeta \quad \text { and } \quad I_{g} f(z)=\int_{0}^{z} f^{\prime}(\zeta) g(\zeta) d \zeta,
$$

where $z \in \mathbb{D}$ and $f \in H(\mathbb{D})$. These operators are close companions of the multiplication operator $M_{g} f(z)=g(z) f(z)$. To see this, note that integration by parts gives

$$
M_{g} f=f(0) g(0)+J_{g} f+I_{g} f .
$$

The boundedness and compactness of $J_{g}$ and $I_{g}$ have recently been characterized on certain spaces of analytic functions. For example, the boundedness of $J_{g}$ on Hardy spaces, Bergman spaces, BMOA space (the space of analytical functions with bounded mean oscillation), and the Möbius invariant space $Q_{p}$, are characterized in $[1,2,6,10]$, respectively.

Next, we write $T_{\varphi, g}$ and $S_{\varphi, g}$ for the operators $C_{\varphi} J_{g}-J_{g} C_{\varphi}$ and $C_{\varphi} I_{g}-I_{g} C_{\varphi}$, respectively. These operators are from $H(p, q, \gamma)$ to $\mathcal{B}^{\alpha}$. Generally speaking, it is clear that $C_{\varphi} J_{g} \neq J_{g} C_{\varphi}$, but it is interesting to consider when

$$
V_{g} C_{\varphi}\left(H_{p, q, \gamma} \rightarrow H_{p, q, \gamma}\right) \equiv C_{\varphi}\left(\mathcal{B}^{\alpha} \rightarrow \mathcal{B}^{\alpha}\right) V_{g} \quad \bmod \mathcal{K},
$$

where $\mathcal{K}$ denotes the collection of all compact operators from $H_{p, q, \gamma}$ to $\mathcal{B}^{\alpha}$, and $V_{g}$ is $J_{g}$ or $I_{g}$. If $C_{\varphi}$ and $V_{g}$ satisfy (1.1), we say they are essentially commutative.

Since the two occurrences of $C_{\varphi}$ in (1.1) act on different spaces, they are in fact different operators. To describe this type of essential commutation more precisely, we say that $C_{\varphi}$ can be transferred from $H(p, q, \gamma)$ to $\mathcal{B}^{\alpha}$ by intertwining $V_{g}$. When it is convenient to de-emphasize the analytic function spaces, we say that $C_{\varphi}$ is $V_{g}$-transferable.

What properties does a nonconstant $g$ have, while (1.1) holds for all $\varphi \in S(\mathbb{D})$ ? An interesting problem is to determine a class of universal symbol $g$ of $V_{g}$ which ensures that all bounded composition operators are $V_{g}$-transferable. Let $X$ and $Y$ be Banach spaces of analytic functions on $\mathbb{D}$, and let $V_{g}: X \rightarrow Y$ be a bounded integraltype operator. The collection of composition operators $C_{\varphi}$ which are bounded both on $X$ and $Y$ is denoted by $\mathscr{B}(X, Y)$.

Definition 1.1 . We denote by $\Omega_{\mathrm{co}}\left(V_{g}\right)$ the collection of all $g$ which satisfy the following two conditions: 
(1) $V_{g}$ is bounded;

(2) every $C_{\varphi} \in \mathscr{B}(X, Y)$ is $V_{g}$-transferable.

We call $\Omega_{\mathrm{co}}\left(V_{g}\right)$ the universal set of $V_{g}$.

Here the subscript 'co' stands for composition operator, while the reference to $V_{g}$ indicates that the universal set is a collection of the symbols of $V_{g}$.

In this paper, we investigate the boundedness and compactness of the operators $C_{\varphi} J_{g}-J_{g} C_{\varphi}$ and $C_{\varphi} I_{g}-I_{g} C_{\varphi}$ from $H(p, q, \gamma)$ to $\mathcal{B}^{\alpha}$. We also describe the universal sets $\Omega_{\text {co }}\left(J_{g}\right)$ and $\Omega_{\text {co }}\left(I_{g}\right)$. Even on the unit disk $\mathbb{D}$ of $\mathbb{C}$, some properties are not easily derived, and we need some new methods and calculation techniques.

Throughout this paper, $C$ denotes a positive constant, the exact value of which will vary from one appearance to the next.

\section{Some lemmas}

To begin our discussion, we state a couple of lemmas which will be used in the proofs of the main results.

The following lemma is the crucial criterion for compactness, and is established by an easy modification of [3, Proof of Proposition 3.11].

Lemma 2.1. Suppose that $0<p, q<\infty, \alpha>0$ and $-1<\gamma<\infty$. Suppose further that $\varphi \in S(\mathbb{D})$ and $g \in H(\mathbb{D})$. Then $T_{\varphi, g}\left(\right.$ respectively $\left.S_{\varphi, g}\right)$ is a compact operator from $H_{p, q, \gamma}$ to $\mathcal{B}^{\alpha}$ if and only if $T_{\varphi, g}$ (respectively $S_{\varphi, g}$ ) is bounded. Also, for any bounded sequence $\left\{f_{k}\right\}_{k \in N}$ in $H_{p, q, \gamma}$ which converges to zero uniformly on compact subsets of $\mathbb{D}$ as $k \rightarrow \infty$, we have $\left\|T_{\varphi, g} f_{k}\right\|_{\mathcal{B}^{\alpha}} \rightarrow 0$ (respectively $\left\|S_{\varphi, g} f_{k}\right\|_{\mathcal{B}^{\alpha}} \rightarrow 0$ ) as $k \rightarrow \infty$.

We set $\beta=(1 / p)+((\gamma+1) / q)$.

Lemma 2.2 [5, Lemma 1]. Assume that $0<p, q<\infty,-1<\gamma<\infty$ and $f \in H_{p, q, \gamma}$. Then there exists a positive constant $C$, independent of $f$, such that

$$
\left|f^{(n)}(z)\right| \leq C \frac{\|f\|_{H_{p, q, \gamma}}}{\left(1-|z|^{2}\right)^{\beta+n}} .
$$

The next lemma holds by direct calculation, and we omit the proof here.

Lemma 2.3. Assume that $0<p, q<\infty, \alpha>0,-1<\gamma<\infty$ and $g \in H(\mathbb{D})$. Then $J_{g}$ : $H(p, q, \gamma) \rightarrow \mathcal{B}^{\alpha}$ is bounded if and only if

$$
\sup _{z \in \mathbb{D}}\left(1-|z|^{2}\right)^{\alpha-\beta}\left|g^{\prime}(z)\right|<\infty,
$$

and $I_{g}$ is bounded if and only if

$$
\sup _{z \in \mathbb{D}}\left(1-|z|^{2}\right)^{\alpha-\beta-1}|g(z)|<\infty .
$$


Lemma 2.4 ([4], or [11, Theorem 1]). Assume that $\varphi \in S(\mathbb{D})$. Then for $\alpha>0, C_{\varphi}$ is bounded on $\mathcal{B}^{\alpha}$ if and only if

$$
\sup _{z \in \mathbb{D}} \frac{\left(1-|z|^{2}\right)^{\alpha}}{\left(1-|\varphi(z)|^{2}\right)^{\alpha}}\left|\varphi^{\prime}(z)\right|<\infty .
$$

Lemma 2.5. Suppose that $\varphi \in S(\mathbb{D})$ and $\varphi(0)=0$. Then $C_{\varphi}$ is bounded on $H_{p, q, \gamma}$.

Proof. By the Littlewood subordination theorem [3, Theorem 2.22],

$$
\begin{aligned}
M_{p}\left(C_{\varphi} f, r\right) & =\left(\frac{1}{2 \pi} \int_{0}^{2 \pi}\left|f\left(\varphi\left(r e^{i \theta}\right)\right)\right|^{p} d \theta\right)^{1 / p} \\
& \leq\left(\frac{1}{2 \pi} \int_{0}^{2 \pi}\left|f\left(r e^{i \theta}\right)\right|^{p} d \theta\right)^{1 / p}=M_{p}(f, r) .
\end{aligned}
$$

Thus

$$
\begin{aligned}
\left\|C_{\varphi} f\right\|_{H_{p, q, \gamma}} & =\left(\int_{0}^{1} M_{p}^{q}\left(C_{\varphi} f, r\right)(1-r)^{\gamma} d r\right)^{1 / q} \\
& \leq\left(\int_{0}^{1} M_{p}^{q}(f, r)(1-r)^{\gamma} d r\right)^{1 / q}=\|f\|_{H_{p, q, \gamma} .}
\end{aligned}
$$

This completes the proof.

\section{Essential commutativity of $C_{\varphi}$ and $J_{g}$ from $H(p, q, \gamma)$ to $\mathcal{B}^{\alpha}$}

In this section, we characterize essential commutativity of the integral operator $J_{g}$ and composition operators from $H_{p, q, \gamma}$ to $\mathcal{B}^{\alpha}$.

Theorem 3.1. Assume that $0<p, q<\infty, \alpha>0$ and $-1<\gamma<\infty$. Assume further that $\varphi \in S(\mathbb{D})$ and $g \in H(\mathbb{D})$. Then $T_{\varphi, g}=C_{\varphi} J_{g}-J_{g} C_{\varphi}$ is a bounded operator from $H_{p, q, \gamma}$ to $\mathcal{B}^{\alpha}$ if and only if

$$
\sup _{z \in \mathbb{D}} \frac{\left(1-|z|^{2}\right)^{\alpha}}{\left(1-|\varphi(z)|^{2}\right)^{\beta}}\left|(g \circ \varphi)^{\prime}(z)-g^{\prime}(z)\right|<\infty .
$$

Proof. Suppose that (3.1) is true. Then for any $f \in H(p, q, \gamma)$,

$$
\begin{aligned}
T_{\varphi, g} f(z) & =C_{\varphi}\left(\int_{0}^{z} f(w) g^{\prime}(w) d w\right)-J_{g}(f \circ \varphi)(z) \\
& =\int_{0}^{\varphi(z)} f(w) g^{\prime}(w) d w-\int_{0}^{z} f(\varphi(w)) g^{\prime}(w) d w .
\end{aligned}
$$

It follows from Lemma 2.2 that

$$
\begin{aligned}
\left\|T_{\varphi, g} f\right\|_{\mathcal{B}^{\alpha}} & =\left|T_{\varphi, g} f(0)\right|+\sup _{z \in \mathbb{D}}\left(1-|z|^{2}\right)^{\alpha}\left|(g \circ \varphi)^{\prime}(z)-g^{\prime}(z)\right||f(\varphi(z))| \\
& \leq C \sup _{z \in \mathbb{D}} \frac{\left(1-|z|^{2}\right)^{\alpha}\left|(g \circ \varphi)^{\prime}(z)-g^{\prime}(z)\right|}{\left(1-|\varphi(z)|^{2}\right)^{\beta}}\|f\|_{H_{p, q, \gamma}} \\
& \leq C\|f\|_{H_{p, q, \gamma},}
\end{aligned}
$$


where the last inequality follows from (3.1). This means that $T_{\varphi, g}=C_{\varphi} J_{g}-J_{g} C_{\varphi}$ is a bounded operator from $H_{p, q, \gamma}$ to $\mathcal{B}^{\alpha}$.

Conversely, suppose that $T_{\varphi, g}: H_{p, q, \gamma} \rightarrow \mathcal{B}^{\alpha}$ is bounded, so that there exists a constant $C$ such that $\left\|T_{\varphi, g} f\right\|_{\mathcal{B}^{\alpha}} \leq C\|f\|_{H_{p, q, \gamma}}$ for any $f \in H_{p, q, \gamma}$.

Now, for any fixed $w \in \mathbb{D}$, we choose the test function

$$
f_{w}(z)=\left(\frac{1-|w|^{2}}{(1-\bar{w} z)^{2}}\right)^{\beta}, \quad z \in \mathbb{D} .
$$

It is straightforward to show that $f_{w} \in H_{p, q, \gamma}$ and $\sup _{w \in \mathbb{D}}\left\|f_{w}\right\|_{H_{p, q, \gamma}}<C$.

Therefore

$$
\begin{aligned}
\infty & >C\left\|T_{\varphi, g}\right\|_{H_{p, q, \gamma} \rightarrow \mathcal{B}^{\alpha}} \geq\left\|T_{\varphi, g} f_{\varphi(\lambda)}\right\|_{\mathcal{B}^{\alpha}} \\
& \geq \sup _{\lambda \in \mathbb{D}}\left(1-|\lambda|^{2}\right)^{\alpha}\left|(g \circ \varphi)^{\prime}(\lambda)-g^{\prime}(\lambda)\right|\left|f_{\varphi(\lambda)}(\varphi(\lambda))\right| \\
& =\sup _{\lambda \in \mathbb{D}}\left(1-|\lambda|^{2}\right)^{\alpha}\left|(g \circ \varphi)^{\prime}(\lambda)-g^{\prime}(\lambda)\right| \frac{1}{\left(1-|\varphi(\lambda)|^{2}\right)^{\beta}},
\end{aligned}
$$

from which the desired result (3.1) holds. This completes the proof.

Theorem 3.2. Assume that $0<p, q<\infty, \alpha>0$ and $-1<\gamma<\infty$. Assume further that $\varphi \in S(\mathbb{D}), g \in H(\mathbb{D})$ and $T_{\varphi, g}=C_{\varphi} J_{g}-J_{g} C_{\varphi}: H_{p, q, \gamma} \rightarrow \mathcal{B}^{\alpha}$. Then $C_{\varphi}$ and $J_{g}$ are essentially commutative if and only if $T_{\varphi, g}$ is bounded and

$$
\lim _{|\varphi(z)| \rightarrow 1} \frac{\left(1-|z|^{2}\right)^{\alpha}}{\left(1-|\varphi(z)|^{2}\right)^{\beta}}\left|(g \circ \varphi)^{\prime}(z)-g^{\prime}(z)\right|=0 .
$$

Proof. Suppose first that $C_{\varphi}$ and $J_{g}$ are essentially commutative. Then $T_{\varphi, g}=C_{\varphi} J_{g}-$ $J_{g} C_{\varphi}$ is compact, hence bounded. Let $\left\{z_{k}\right\}_{k \in N}$ be a sequence in $\mathbb{D}$ such that $\left|\varphi\left(z_{k}\right)\right| \rightarrow 1$ as $k \rightarrow \infty$. We consider the test functions defined by

$$
f_{k}(z)=\left(\frac{1-\left|\varphi\left(z_{k}\right)\right|^{2}}{\left(1-\overline{\varphi\left(z_{k}\right)} z\right)^{2}}\right)^{\beta} .
$$

Direct computation shows that $f_{k} \in H_{p, q, \gamma}$ with $\sup _{k}\left\|f_{k}\right\|_{H_{p, q, \gamma}}<C$ and $\left\{f_{k}\right\}$ converges to 0 uniformly on compact subsets of $\mathbb{D}$ as $k \rightarrow \infty$. Since $T_{\varphi, g}=C_{\varphi} J_{g}-J_{g} C_{\varphi}$ is compact, it follows from Lemma 2.1 that

$$
\lim _{k \rightarrow \infty}\left\|T_{\varphi, g} f_{k}\right\|_{\mathcal{B}^{\alpha}}=0 .
$$

Therefore

$$
\begin{aligned}
\left\|T_{\varphi, g} f_{k}\right\|_{\mathcal{B}^{\alpha}} & \geq\left(1-\left|z_{k}\right|^{2}\right)^{\alpha}\left|(g \circ \varphi)^{\prime}\left(z_{k}\right)-g^{\prime}\left(z_{k}\right)\right|\left|f_{k}\left(\varphi\left(z_{k}\right)\right)\right| \\
& =\left(1-\left|z_{k}\right|^{2}\right)^{\alpha}\left|(g \circ \varphi)^{\prime}\left(z_{k}\right)-g^{\prime}\left(z_{k}\right)\right| \frac{1}{\left(1-\left|\varphi\left(z_{k}\right)\right|^{2}\right)^{\beta}} .
\end{aligned}
$$

Combining (3.5) and (3.6), the condition (3.3) follows. 
Conversely, suppose that $T_{\varphi, g}$ is bounded and (3.3) holds. By Theorem 3.1, the boundedness of $T_{\varphi, g}$ implies that

$$
\left(1-|z|^{2}\right)^{\alpha}\left|(g \circ \varphi)^{\prime}(z)-g^{\prime}(z)\right| \leq C
$$

for any $z \in \mathbb{D}$.

Let $\left\{f_{k}\right\}_{k \in N}$ be any bounded sequence in $H_{p, q, \gamma}$ which converges to zero uniformly on compact subsets of $\mathbb{D}$ as $k \rightarrow \infty$, with $\sup _{k}\left\|f_{k}\right\|_{H_{p, q, \gamma}} \leq M$.

It follows from (3.3) that for any $\varepsilon>0$, there exists $\delta>0$, with $\delta<|\varphi(z)|<1$, such that

$$
\frac{\left(1-|z|^{2}\right)^{\alpha}}{\left(1-|\varphi(z)|^{2}\right)^{\beta}}\left|(g \circ \varphi)^{\prime}(z)-g^{\prime}(z)\right|<\frac{\varepsilon}{M} .
$$

Let $E=\{z \in \mathbb{D}:|\varphi(z)| \leq \delta\}$, and let $K=\{w=\varphi(z): z \in E\}$, a compact subset of $\mathbb{D}$. By Theorem 3.1, the boundedness of $T_{\varphi, g}$ implies (3.1), and it follows from (3.7) and (3.8) that

$$
\begin{aligned}
&\left\|T_{\varphi, g} f_{k}\right\|_{\mathcal{B}^{\alpha}} \leq\left|T_{\varphi, g} f_{k}(0)\right|+\sup _{z \in E}\left(1-|z|^{2}\right)^{\alpha}\left|(g \circ \varphi)^{\prime}(z)-g^{\prime}(z)\right|\left|f_{k}(\varphi(z))\right| \\
& \quad+\sup _{z \in \mathbb{D} \backslash E}\left(1-|z|^{2}\right)^{\alpha}\left|(g \circ \varphi)^{\prime}(z)-g^{\prime}(z)\right| \frac{\left\|f_{k}\right\|_{H_{p, q, \gamma}}}{\left(1-|\varphi(z)|^{2}\right)^{\beta}} \\
& \leq\left|T_{\varphi, g} f_{k}(0)\right|+C \sup _{w \in K}\left|f_{k}(w)\right|+\varepsilon .
\end{aligned}
$$

Note that $\varepsilon$ is an arbitrary positive number. In view of the assumption that $f_{k} \rightarrow 0$ as $k \rightarrow \infty$ on compact subsets of $\mathbb{D}$, on letting $k \rightarrow \infty$ in (3.9), we have $\left\|T_{\varphi, g} f_{k}\right\|_{\mathcal{B}^{\alpha}} \rightarrow 0$. It follows from Lemma 2.1 that $T_{\varphi, g}$ is compact, and so $C_{\varphi}$ and $J_{g}$ are essentially commutative. The proof of the theorem is complete.

Theorem 3.3. Assume that $0<p, q<\infty, \alpha>0,-1<\gamma<\infty, \varphi \in S(\mathbb{D})$ and $g \in H(\mathbb{D})$. Assume further that $J_{g}: H(p, q, \gamma) \rightarrow \mathcal{B}^{\alpha}$ is bounded. Then $J_{g}$ is essentially commutative with any $C_{\varphi} \in \mathscr{B}\left(H_{p, q, \gamma}, \mathcal{B}^{\alpha}\right)$ if and only if

$$
\lim _{|z| \rightarrow 1}\left(1-|z|^{2}\right)^{\alpha-\beta}\left|g^{\prime}(z)\right|=0
$$

that is, $\Omega_{\mathrm{co}}\left(J_{g}\right)=\mathcal{B}_{0}^{\alpha-\beta}$.

Proof. We first prove necessity. Let $\varphi(z)=e^{i \theta} z$ for any $\theta \in[0,2 \pi]$. Then by Lemmas 2.4 and 2.5 , it is easy to see that $C_{\varphi}$ is bounded both on $H_{p, q, \gamma}$ and $\mathcal{B}^{\alpha}$, so that $C_{\varphi} \in \mathscr{B}\left(H_{p, q, \gamma}, \mathcal{B}^{\alpha}\right)$. From the assumptions we know that $J_{g}$ and $C_{\varphi}$ are essentially commutative, and it follows from Theorem 3.2 that

$$
\lim _{|z| \rightarrow 1}\left(1-|z|^{2}\right)^{\alpha-\beta}\left|e^{i \theta} g^{\prime}\left(e^{i \theta} z\right)-g^{\prime}(z)\right|=0 .
$$

It is necessary to bound from above the left-hand side of (3.11). We have

$$
\begin{aligned}
\left(1-|z|^{2}\right)^{\alpha-\beta}\left|e^{i \theta} g^{\prime}\left(e^{i \theta} z\right)-g^{\prime}(z)\right| & \leq\left(1-|z|^{2}\right)^{\alpha-\beta}\left|e^{i \theta} g^{\prime}\left(e^{i \theta} z\right)\right|+\left(1-|z|^{2}\right)^{\alpha-\beta}\left|g^{\prime}(z)\right| \\
& =\left(1-\left|e^{i \theta} z\right|^{2}\right)^{\alpha-\beta}\left|g^{\prime}\left(e^{i \theta} z\right)\right|+\left(1-|z|^{2}\right)^{\alpha-\beta}\left|g^{\prime}(z)\right| \\
& \leq 2\|g\|_{\mathcal{B}^{\alpha-\beta}} .
\end{aligned}
$$


By Lemma 2.2, the boundedness of $J_{g}: H(p, q, \gamma) \rightarrow \mathcal{B}^{\alpha}$ implies that $\|g\|_{\mathcal{B}^{\alpha-\beta}}$ is finite, and so the left-hand side of (3.11) is bounded independently of $\theta$. We write $g(z)=$ $\sum_{n=0}^{\infty} a_{n} z^{n}$, and integrate the left-hand side of (3.11) with respect to $\theta$ from 0 to $2 \pi$. By the dominating convergence theorem, we deduce that

$$
\begin{aligned}
0 & =\int_{0}^{2 \pi} \lim _{|z| \rightarrow 1}\left(1-|z|^{2}\right)^{\alpha-\beta}\left|e^{i \theta} g^{\prime}\left(e^{i \theta} z\right)-g^{\prime}(z)\right| d \theta \\
& =\lim _{|z| \rightarrow 1} \int_{0}^{2 \pi}\left(1-|z|^{2}\right)^{\alpha-\beta}\left|\sum_{n=1}^{\infty} n a_{n} z^{n-1}\left(e^{i n \theta}-1\right)\right| d \theta \\
& \geq \lim _{|z| \rightarrow 1}\left(1-|z|^{2}\right)^{\alpha-\beta}\left|\sum_{n=1}^{\infty} n a_{n} z^{n-1} \int_{0}^{2 \pi}\left(e^{i n \theta}-1\right) d \theta\right| \geq \lim _{|z| \rightarrow 1}\left(1-|z|^{2}\right)^{\alpha-\beta}\left|g^{\prime}(z)\right| .
\end{aligned}
$$

Hence (3.10) holds, and so $g \in \mathcal{B}_{0}^{\alpha-\beta}$.

Turning to sufficiency, suppose that (3.10) holds. Corollary 2.40 in [3] states that $|\varphi(z)| \leq(|z|+|\varphi(0)|) /(1+|z||\varphi(0)|)$, and so we have

$$
\frac{1-|z|}{1-|\varphi(z)|} \leq \frac{1+|z||\varphi(0)|}{1-|\varphi(0)|}
$$

By Lemmas 2.4, 2.5 and (3.12),

$$
\begin{aligned}
\lim _{|\varphi(z)| \rightarrow 1} & \frac{\left(1-|z|^{2}\right)^{\alpha}}{\left(1-|\varphi(z)|^{2}\right)^{\beta}}\left|(g \circ \varphi)^{\prime}(z)-g^{\prime}(z)\right| \\
\leq & \lim _{|\varphi(z)| \rightarrow 1} \frac{\left(1-|z|^{2}\right)^{\alpha}}{\left(1-|\varphi(z)|^{2}\right)^{\alpha}}\left|\varphi^{\prime}(z)\right|\left(1-|\varphi(z)|^{2}\right)^{\alpha-\beta}\left|g^{\prime}(\varphi(z))\right| \\
& +\lim _{|\varphi(z)| \rightarrow 1} \frac{(2(1-|z|))^{\alpha}}{(1-|\varphi(z)|)^{\alpha}}\left(1-|z|^{2}\right)^{\alpha-\beta}\left|g^{\prime}(z)\right| \\
\leq & C \lim _{|\varphi(z)| \rightarrow 1}\left(1-|\varphi(z)|^{2}\right)^{\alpha-\beta}\left|g^{\prime}(\varphi(z))\right| \\
& +2^{\alpha} \lim _{|\varphi(z)| \rightarrow 1}\left(\frac{1+|z||\varphi(0)|}{1-|\varphi(0)|}\right)^{\alpha}\left(1-|z|^{2}\right)^{\alpha-\beta}\left|g^{\prime}(z)\right| \\
\leq & C \lim _{|w| \rightarrow 1}\left(1-|w|^{2}\right)^{\alpha-\beta}\left|g^{\prime}(w)\right|+C \lim _{|z| \rightarrow 1}\left(1-|z|^{2}\right)^{\alpha-\beta}\left|g^{\prime}(z)\right|=0 .
\end{aligned}
$$

By Theorem 3.2, $J_{g}$ is essentially commutative with any $C_{\varphi} \in \mathscr{B}\left(H_{p, q, \gamma}, \mathcal{B}^{\alpha}\right)$, so that $g \in \Omega_{\mathrm{co}}\left(J_{g}\right)$. This completes the proof.

\section{Essential commutativity of $I_{g}$ and $C_{\varphi}$ from $H_{p, q, \gamma}$ to $\mathcal{B}^{\alpha}$}

We characterize essential commutativity of the integral operator $I_{g}$ and composition operators from $H_{p, q, \gamma}$ to $\mathcal{B}^{\alpha}$ in this section. 
Theorem 4.1. Assume that $0<p, q<\infty, \alpha>0$ and $-1<\gamma<\infty$. Assume further that $\varphi \in S(\mathbb{D})$ and $g \in H(\mathbb{D})$. Then $S_{\varphi, g}=C_{\varphi} I_{g}-I g C_{\varphi}$ is a bounded operator from $H(p, q, \gamma)$ to $\mathcal{B}^{\alpha}$ if and only if

$$
\sup _{z \in \mathbb{D}} \frac{\left(1-|z|^{2}\right)^{\alpha}}{\left(1-|\varphi(z)|^{2}\right)^{\beta+1}}\left|\varphi^{\prime}(z)\right||g(\varphi(z))-g(z)|<\infty .
$$

Proof. First, suppose that $S_{\varphi, g}: H_{p, q, \gamma} \rightarrow \mathcal{B}^{\alpha}$ is bounded, so that there exists a constant $C$ such that $\left\|S_{\varphi, g} f\right\|_{\mathcal{B}^{\alpha}} \leq C\|f\|_{H_{p, q, \gamma}}$ for all $f \in H_{p, q, \gamma}$. Note that

$$
\left(1-|z|^{2}\right)^{\alpha}\left|\left(S_{\varphi, g} f\right)^{\prime}(z)\right|=\left(1-|z|^{2}\right)^{\alpha}\left|\varphi^{\prime}(z)\right||g(\varphi(z))-g(z)|\left|f^{\prime}(\varphi(z))\right| .
$$

Taking $f(z)=z$, we deduce that

$$
\sup _{z \in \mathbb{D}}\left(1-|z|^{2}\right)^{\alpha}\left|\varphi^{\prime}(z)\right||g(\varphi(z))-g(z)|<\infty .
$$

We now choose the same test function (3.2), and let $\lambda \in \mathbb{D}$. Then one can show that

$$
\left|f_{\varphi(\lambda)}^{\prime}(\varphi(\lambda))\right|=2\left(\frac{1}{p}+\frac{\gamma+1}{q}\right) \frac{|\varphi(\lambda)|}{\left(1-|\varphi(\lambda)|^{2}\right)^{\beta+1}} .
$$

Therefore

$$
\begin{aligned}
\infty & >C\left\|S_{\varphi, g}\right\|_{H_{p, q, \gamma} \rightarrow \mathcal{B}^{\alpha}} \geq\left\|S_{\varphi, g} f_{\varphi(\lambda)}\right\|_{\mathcal{B}^{\alpha}} \\
& \geq 2\left(\frac{1}{p}+\frac{\gamma+1}{q}\right) \frac{\left(1-|\lambda|^{2}\right)^{\alpha}|\varphi(\lambda)|\left|\varphi^{\prime}(z)\right|}{\left(1-|\varphi(\lambda)|^{2}\right)^{\beta+1}}|g(\varphi(\lambda))-g(\lambda)|,
\end{aligned}
$$

from which we obtain

$$
\begin{aligned}
\sup _{|\varphi(\lambda)|>1 / 2} & \frac{\left(1-|\lambda|^{2}\right)^{\alpha}\left|\varphi^{\prime}(\lambda)\right|}{\left(1-|\varphi(\lambda)|^{2}\right)^{\beta+1}}|g(\varphi(\lambda))-g(\lambda)| \\
\quad \leq & \sup _{|\varphi(\lambda)|>1 / 2} 2 \frac{\left(1-|\lambda|^{2}\right)^{\alpha}|\varphi(\lambda)|\left|\varphi^{\prime}(\lambda)\right|}{\left(1-|\varphi(\lambda)|^{2}\right)^{\beta+1}}|g(\varphi(\lambda))-g(\lambda)|<\infty .
\end{aligned}
$$

It follows from (4.2) that

$$
\begin{aligned}
\sup _{|\varphi(\lambda)| \leq 1 / 2} & \frac{\left(1-|\lambda|^{2}\right)^{\alpha}\left|\varphi^{\prime}(\lambda)\right|}{\left(1-|\varphi(\lambda)|^{2}\right)^{\beta+1}}|g(\varphi(\lambda))-g(\lambda)| \\
\quad \leq & \sup _{|\varphi(\lambda)| \leq 1 / 2}\left(\frac{4}{3}\right)^{\beta+1}\left(1-|\lambda|^{2}\right)^{\alpha}\left|\varphi^{\prime}(\lambda)\right||g(\varphi(\lambda))-g(\lambda)|<\infty .
\end{aligned}
$$

Combining (4.3) and (4.4), the desired result (4.1) follows.

Conversely, suppose that the condition (4.1) holds. Then for any $f \in H_{p, q, \gamma}$, we deduce from Lemma 2.2 that

$$
\begin{aligned}
\left\|S_{\varphi, g} f\right\|_{\mathcal{B}^{\alpha}} & =\left|S_{\varphi, g} f(0)\right|+\sup _{z \in D}\left(1-|z|^{2}\right)^{\alpha}\left|\varphi^{\prime}(z)\right||g(\varphi(z))-g(z)|\left|f^{\prime}(\varphi(z))\right| \\
& \leq C \sup _{z \in D} \frac{\left(1-|z|^{2}\right)^{\alpha}\left|\varphi^{\prime}(z)\right|}{\left(1-|\varphi(z)|^{2}\right)^{\beta+1}}|g(\varphi(z))-g(z)|\|f\|_{H_{p, q, \gamma}} \\
& \leq C\|f\|_{H_{p, q, \gamma} .}
\end{aligned}
$$

Hence $S_{\varphi, g}=C_{\varphi} I_{g}-I_{g} C_{\varphi}$ is bounded from $H_{p, q, \gamma}$ to $\mathcal{B}^{\alpha}$. This completes the proof. 
Theorem 4.2. Assume that $0<p, q<\infty, \alpha>0,-1<\gamma<\infty, \varphi \in S(\mathbb{D})$ and $g \in H(\mathbb{D})$. Assume further that $S_{\varphi, g}=C_{\varphi} I_{g}-I_{g} C_{\varphi}: H_{p, q, \gamma} \rightarrow \mathcal{B}^{\alpha}$. Then $C_{\varphi}$ and $I_{g}$ are essentially commutative if and only if $S_{\varphi, g}$ is bounded and

$$
\lim _{|\varphi(z)| \rightarrow 1} \frac{\left(1-|z|^{2}\right)^{\alpha}}{\left(1-|\varphi(z)|^{2}\right)^{\beta+1}}\left|\varphi^{\prime}(z)\right||g(\varphi(z))-g(z)|=0 .
$$

Proof. We first prove sufficiency. For any bounded sequence $\left\{f_{k}\right\}_{k \in N}$ in $H_{p, q, \gamma}$ which converges to zero uniformly on compact subsets of $\mathbb{D}$ as $k \rightarrow \infty$, we have $\sup _{k}\left\|f_{k}\right\|_{H_{p, q, \gamma}} \leq M$. From (4.5), there exists $\delta>0, \delta<|\varphi(z)|<1$, such that for any $\varepsilon>0$,

$$
\frac{\left(1-|z|^{2}\right)^{\alpha}\left|\varphi^{\prime}(z)\right|}{\left(1-|\varphi(z)|^{2}\right)^{\beta+1}}|g(\varphi(z))-g(z)|<\frac{\varepsilon}{M} .
$$

Let $E=\{z \in \mathbb{D}:|\varphi(z)| \leq \delta\}$, and let $K=\{w=\varphi(z): z \in E\}$, a compact subset of $\mathbb{D}$. By the boundedness of $S_{\varphi, g}$, it follows from (4.2) and (4.6) that

$$
\begin{aligned}
&\left\|S_{\varphi, g} f_{k}\right\|_{\mathcal{B}^{\alpha}} \leq\left|S_{\varphi, g} f_{k}(0)\right|+\sup _{z \in E}\left(1-|z|^{2}\right)^{\alpha}\left|\varphi^{\prime}(z)\right||g(\varphi(z))-g(z)|\left|f_{k}^{\prime}(\varphi(z))\right| \\
& \quad+\sup _{z \in \mathbb{D} \backslash E} \frac{\left(1-|z|^{2}\right)^{\alpha}\left|\varphi^{\prime}(z)\right|}{\left(1-|\varphi(z)|^{2}\right)^{\beta+1}}|g(\varphi(z))-g(z)|\left\|f_{k}\right\|_{H_{p, q, \gamma}} \\
& \leq\left|S_{\varphi, g} f_{k}(0)\right|+C \sup _{w \in K}\left|f_{k}^{\prime}(w)\right|+\varepsilon .
\end{aligned}
$$

The assumption that $f_{k} \rightarrow 0$ as $k \rightarrow \infty$ on compact subsets of $\mathbb{D}$, along with Cauchy's estimate, gives that $f_{k}^{\prime} \rightarrow 0$ as $k \rightarrow \infty$ on compact subsets of $\mathbb{D}$. Letting $k \rightarrow \infty$ in (4.7), and noting that $\varepsilon$ is an arbitrary positive number, we have $\left\|S_{\varphi, g} f_{k}\right\|_{\mathcal{B}^{\alpha}} \rightarrow 0$. Then it follows from Lemma 2.1 that $C_{\varphi}$ and $I_{g}$ are essentially commutative.

We now turn to necessity. First, suppose that $C_{\varphi}$ and $I_{g}$ are essentially commutative. Then $S_{\varphi, g}=C_{\varphi} I_{g}-I_{g} C_{\varphi}$ is compact, hence bounded. Let $\left\{z_{k}\right\}_{k \in N}$ be a sequence in $\mathbb{D}$ such that $\left|\varphi\left(z_{k}\right)\right| \rightarrow 1$ as $k \rightarrow \infty$, and consider the sequence $\left\{f_{k}\right\}_{k \in N}$ defined by (3.4) as in the proof of Theorem 3.2. Then one can show that

$$
\left|f_{k}^{\prime}\left(\varphi\left(z_{k}\right)\right)\right|=2\left(\frac{1}{p}+\frac{\gamma+1}{q}\right) \frac{\left|\varphi\left(z_{k}\right)\right|}{\left(1-\left|\varphi\left(z_{k}\right)\right|^{2}\right)^{\beta+1}} .
$$

Thus

$$
\begin{aligned}
\left\|S_{\varphi, g} f_{k} \mid\right\|_{\mathcal{B}^{\alpha}} & \geq\left(1-\left|z_{k}\right|^{2}\right)^{1-\alpha}\left|\varphi^{\prime}\left(z_{k}\right)\right|\left|g\left(\varphi\left(z_{k}\right)\right)-g\left(z_{k}\right)\right|\left|f_{k}^{\prime}\left(\varphi\left(z_{k}\right)\right)\right| \\
& =2\left(\frac{1}{p}+\frac{\gamma+1}{q}\right) \frac{\left|\varphi\left(z_{k}\right)\right|\left(1-\left|z_{k}\right|^{2}\right)^{1-\alpha}\left|\varphi^{\prime}\left(z_{k}\right)\right|\left|g\left(\varphi\left(z_{k}\right)\right)-g\left(z_{k}\right)\right|}{\left(1-\left|\varphi\left(z_{k}\right)\right|^{2}\right)^{\beta+1}} .
\end{aligned}
$$

Since $S_{\varphi, g}=C_{\varphi} I_{g}-I_{g} C_{\varphi}$ is compact, by Lemma 2.1 we have $\lim _{k \rightarrow \infty}\left\|S_{\varphi, g} f_{k}\right\|_{\mathcal{B}}^{\alpha}=0$. Combining this with (4.8), and noting that the right-hand side of (4.8) tends to zero as $k \rightarrow \infty$, the desired result (4.5) follows. The proof is complete. 
Theorem 4.3. Assume that $0<p, q<\infty, 0<\alpha<1,-1<\gamma<\infty, \varphi \in S(\mathbb{D})$ and $g \in$ $H(\mathbb{D})$. Assume further that $I_{g}: H(p, q, \gamma) \rightarrow \mathcal{B}^{\alpha}$ is bounded. Then $I_{g}$ is essentially commutative with any $C_{\varphi} \in \mathscr{B}\left(H_{p, q, \gamma}, \mathcal{B}^{\alpha}\right)$ if and only if

$$
\lim _{|z| \rightarrow 1}\left(1-|z|^{2}\right)^{\alpha-\beta-1}|g(z)|=0,
$$

that is, $\Omega_{\mathrm{co}}\left(I_{g}\right)=\mathcal{B}_{0}^{\alpha-\beta-1}$.

Proof. The proof is similar to that of Theorem 3.3, and we omit the details.

\section{Acknowledgements}

The authors would like to thank the editor Graeme Cohen and the referee for helpful comments and suggestions which improved the presentation of this paper.

\section{References}

[1] A. Aleman and J. A. Cima, 'An integral operator on $H^{p}$ and Hardy's inequality', J. Anal. Math. 85 (2001), 157-176.

[2] A. Aleman and A. G. Siskakis, 'Integration operators on Bergman spaces', Indiana Univ. Math. 46(2) (1997), 337-356.

[3] C. C. Cowen and B. MacCluer, Composition Operators on Spaces of Analytic Functions (CRC Press, Boca Raton, FL, 1995).

[4] S. Li and S. Stević, 'Composition followed by differentiation between Bloch-type spaces', J. Comput. Anal. Appl. 9(2) (2007), 195-205.

[5] S. X. Li and S. Stević, 'Composition followed by differentiation from mixed-norm spaces to $\alpha$-Bloch spaces', Math. Sbornik 199(12) (2008), 117-128.

[6] A. G. Siskakis and R. H. Zhao, 'A Volterra type operator on spaces of analytic functions. Function spaces (Edwardsville, IL, 1998)’, Contemp. Math. 232 (1999), 299-311.

[7] M. Tjani, 'Compact composition operators on Besov spaces', Trans. Amer. Math. Soc. 355(11) (2003), 4683-4698.

[8] Z. Wu, 'Carleson measures and multipliers for Dirichlet spaces', J. Funct. Anal. 169 (1999), 148-163.

[9] H. Wulan, D. Zheng and K. Zhu, 'Compact composition operators on BMOA and the Bloch space', Proc. Amer. Math. Soc. 137 (2009), 3861-3868.

[10] J. Xiao, 'The $Q_{p}$ Carelson measure problem', Adv. in Math. 217 (2008), 2075-2088.

[11] Z. H. Zhou, 'Composition operator on the Lipschitz space in polydiscs', Sci. China Ser. A 46(1) (2003), 33-38.

[12] Z. H. Zhou and R. Y. Chen, 'Weighted composition operators fom $F(p, q, s)$ to Bloch type spaces', Internat. J. Math. 19(8) (2008), 899-926.

[13] Z. H. Zhou and J. H. Shi, 'Compactness of composition operators on the Bloch space in classical bounded symmetric domains', Michigan Math. J. 50 (2002), 381-405.

[14] K. H. Zhu, Spaces of Holomorphic Functions in the Unit Ball, Graduate Texts in Mathematics, 226 (Springer, New York, 2005).

\section{ZE-HUA ZHOU, Department of Mathematics, Tianjin University, Tianjin 300072, PR China e-mail: zehuazhou2003@yahoo.com.cn}


LIANG ZHANG, Department of Mathematics, Tianjin University, Tianjin 300072, PR China

e-mail: 168zhangliang2011@163.com

HONG-GANG ZENG, Department of Mathematics, Tianjin University, Tianjin 300072, PR China

e-mail: zhgng@tju.edu.cn 